\title{
Selectivity for Three-Dimensional Shape and Grasping-Related Activity in the Macaque Ventral Premotor Cortex
}

\author{
Tom Theys, ${ }^{1,2}$ Pierpaolo Pani, ${ }^{1}$ Johannes van Loon, ${ }^{2}$ Jan Goffin, ${ }^{2}$ and Peter Janssen ${ }^{1}$ \\ ${ }^{1}$ Laboratorium voor Neuro- en Psychofysiologie and ${ }^{2}$ Afdeling Experimentele Neurochirurgie en Neuroanatomie, Katholieke Universiteit Leuven, B-3000 \\ Leuven, Belgium
}

\begin{abstract}
Anatomical studies indicate that area F5 in the macaque ventral premotor cortex consists of three different sectors. One of these is F5a in the posterior bank of the inferior arcuate sulcus, but no functional characterization of F5a at the single-cell level exists. We investigated the neuronal selectivity for three-dimensional (3D) shape and grasping activity in F5a. In contrast to neighboring regions F5p and $45 \mathrm{~B}$, the great majority of F5a neurons showed selectivity for disparity-defined curved surfaces, and most neurons preserved this selectivity across positions in depth, indicating higher-order disparity selectivity. Thus, as predicted by monkey fMRI data, F5a neurons showed robust 3D-shape selectivity in the absence of a motor response. To investigate the relationship between disparity selectivity and grasping activity, we recorded from 3D-shape-selective F5a neurons during a visually guided grasping task and during grasping in the dark. F5a neurons encoding the depth profile of curved surfaces frequently responded during grasping of real-world objects in the light, but not in the dark, whereas nearby neurons were also active in the dark. The presence of 3D-shape-selective and "visual-dominant" neurons demonstrates that the F5a sector is distinct from neighboring regions of ventral premotor cortex, in line with recent anatomical connectivity studies.
\end{abstract}

\section{Introduction}

Converting visual three-dimensional (3D) object information into appropriate distal hand motor outputs produces preshaping of the hand for the control of grasping (Sakata et al., 1997; Castiello and Begliomini, 2008). An important depth cue for grasping comes from binocular disparity (Watt and Bradshaw, 2003), which results from the different vantage points of the two eyes.

The ventral premotor cortex (area F5) is considered crucial for prehension in primates (Rizzolatti et al., 1988; Godschalk et al., 1995; Fogassi et al., 2001). Cytoarchitectonically, area F5 consists of F5p caudally, F5c on the convexity, and F5a anteriorly in the posterior bank of the inferior arcuate sulcus (Belmalih et al., 2009). Area F5a also has a distinct anatomical connectivity (Gerbella et al., 2011), characterized by strong connections with the prefrontal cortex (areas 46 and 12), posterior parietal area AIP (anterior intraparietal area), the SII region, and the other adjacent ventral premotor areas, but not with primary motor cortex,

\footnotetext{
Received April 9, 2012; revised June 9, 2012; accepted July 5, 2012

Author contributions: T.T., P.P., J.v.L., J.G., and P.J. designed research;T.T.,P.P., and P.J. performed research; T.T., P.P., and P.J. analyzed data; T.T., P.P., J.v.L., and P.J. wrote the paper.

This work was supported by Geconcerteerde Onderzoeksacties Grants 2005/18 and 2010/19, Geneeskundige Stichting Koningin Elisabeth, Fonds voor Wetenschappelijk Onderzoek Vlaanderen Grants G.0495.05 and G.0713.09, Excellentie financiering Grant EF05/014, and Programma financiering Grant PFV/10/008. We thank Piet Kayenbergh, Gerrit Meulemans, Stijn Verstraeten, Marc Depaep, Wouter Depuydt, Thembi Frank, and Inez Puttemans for technical assistance, Olivier Joly for the $3 \mathrm{D}$ rendering of the arcuate sulcus, and Steve Raiguel for comments on this manuscript.

The authors declare no competing financial interests.

Correspondence should be addressed to Dr. Peter Janssen, Laboratorium voor Neuro- en Psychofysiologie, Leuven Medical School, Herestraat 49, Bus 1021, B-3000 Leuven, Belgium. E-mail: peter.janssen@med.kuleuven.be. DOI:10.1523/JNEUROSCI.1790-12.2012

Copyright $\odot 2012$ the authors $\quad 0270-6474 / 12 / 3212038-13 \$ 15.00 / 0$
}

in contrast to the other F5 subdivisions. This pattern of anatomical connectivity suggests that F5a could be considered a prepremotor area, integrating parietal and prefrontal inputs and projecting to adjacent F5 sectors (Gerbella et al., 2011).

All single-cell recording studies have been conducted in F5c and F5p (Murata et al., 1997; Raos et al., 2006; Townsend et al., 2011). A subclass of these F5 neurons respond selectively to the visual presentation of graspable $3 \mathrm{D}$ objects, and frequently the type of grasping action thus coded corresponds to the shape and size of the object that elicits a response (Jeannerod et al., 1995). To date, however, no single-cell data are available on area F5a.

The only functional evidence for a separate area in the posterior bank of the inferior arcuate sulcus was reported by an awake monkey fMRI study (Joly et al., 2009), showing that F5a was more activated by curved surfaces than by flat surfaces at different positions in depth. Srivastava et al. (2009) showed that a subset of neurons in area AIP encode the depth profile of curved surfaces defined by disparity (i.e., higher-order disparity selective). Since AIP and F5a are strongly interconnected as parts of the parietofrontal grasping circuit (Nelissen and Vanduffel, 2011), and in view of the fMRI results of Joly et al. (2009), we wanted to determine whether individual F5a neurons were indeed higher-order disparity selective, similar to neurons in area AIP (Srivastava et al., 2009) and inferior temporal cortex (IT) (Janssen et al., 1999). Furthermore, we characterized 3D-shape-selective F5a neurons during grasping in the light and in the dark. We found that many F5a neurons exhibited strong selectivity for the depth profile of curved surfaces, and that a large majority of these neurons were active during visually guided grasping but not during grasping in the dark. However, 3D-shape-selective F5a neurons were embedded within clusters of neurons that also fired during grasping in 


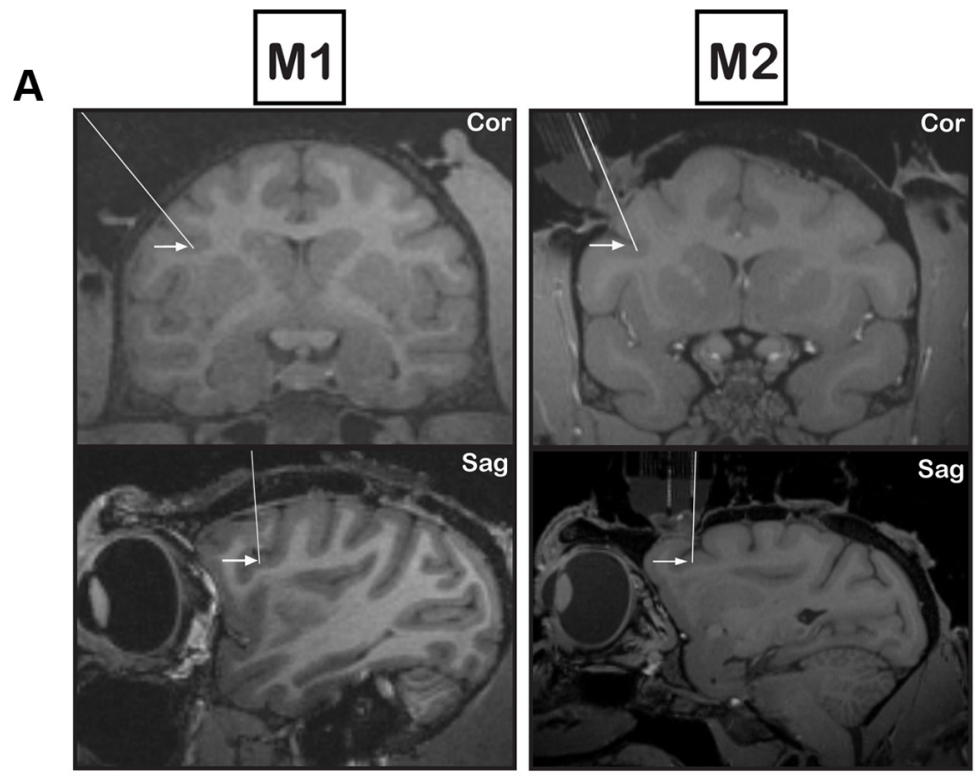

B

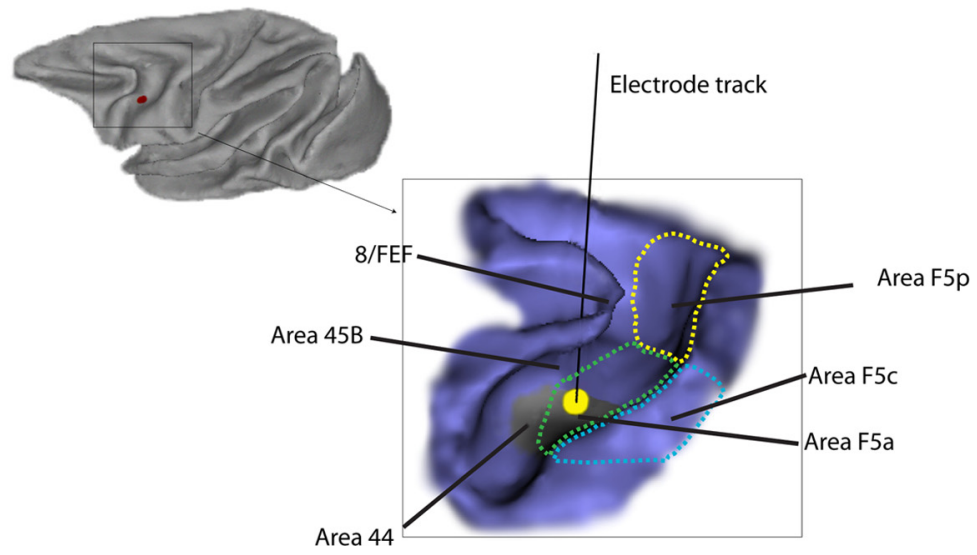

Figure 1. Recording positions in F5a. $\boldsymbol{A}$, Coronal (top row) and sagittal (bottom row) MRI illustrating the most representative recording sites (arrows) and electrode tracks (lines) in the inferior arcuate sulcus in M1 (left) and M2 (right). $\boldsymbol{B}$, Lateral view of the macaque brain and 3D rendering of the arcuate sulcus of M1. The inset shows an electrode penetration in the inferior limb of the arcuate sulcus in the center of the area with higher-order disparity-selective neurons (black line). The electrode track traversed area $45 \mathrm{~B}$ and ended in the lateral bank of the arcuate sulcus (area F5a). The different subsectors of F5 have been indicated on the drawing. The gray area in the lateral bank of the inferior arcuate sulcus indicates the fMRI activation evoked by curved surfaces described by Joly et al. (2009).

the dark (i.e., visuomotor neurons), which may constitute a critical neural circuit for visuomotor transformations. These distinct functional properties combined with cytoarchitectonic and connectivity data support the view that F5a is a separate area in the ventral premotor cortex.

\section{Materials and Methods}

Subjects and recording procedure

Two male rhesus monkeys (M1 and M2) served as subjects for extracellular microelectrode recordings [M2 was also used in the study by Srivastava et al. (2009) on AIP]. The surgical and recording procedures were similar to previous experiments (Janssen et al., 1999, 2000b; Srivastava et al., 2009). Under isoflurane anesthesia, an MRI-compatible head fixation post and recording chamber were implanted using dental acrylic and ceramic screws. We implanted the recording chamber at Horsley-Clarke coordinates $21 \mathrm{~mm}$ anterior and $20 \mathrm{~mm}$ lateral at an angle of $40^{\circ}$ with respect to the vertical for monkey 1 and at an angle of $25^{\circ}$ for monkey 2 , aimed at the depth of the posterior bank of the inferior limb of the arcuate sulcus. In this region of F5a, Joly et al. (2009) reported stronger activation for curved surfaces compared to flat surfaces at dif- ferent positions in depth. All surgical techniques and veterinary care were performed in accordance with the NIH Guide for Care and Use of Laboratory Animals and approved by the local ethical committee of the Katholieke Universiteit Leuven.

For extracellular recordings, tungsten microelectrodes (MicroProbes) were inserted through a guide tube placed in a standard grid (Crist Instruments). Magnetic resonance imaging using glass capillaries filled with a $1 \%$ copper sulfate solution inserted into key grid positions confirmed correct positioning of the electrode in the depth of the posterior bank of the inferior arcuate sulcus (area F5a; Fig. 1).

Since our recording positions were situated close to area 44 where orofacial somatomotor responses have been observed (Petrides et al., 2005), we also performed intracortical microstimulation in the center of the recording area with the following parameters: $500 \mathrm{~ms}$ stimulation duration with $0.2 \mathrm{~ms}$ pulsewidth at 200 $\mathrm{Hz}$; stimulation intensity ranged from 30 to $150 \mu \mathrm{A}$ (Graziano et al., 2002).

\section{Testing procedure}

Disparity and position-in-depth test. The animals were trained to perform a passive fixation task, keeping the gaze of both eyes inside a $1^{\circ}$ fixation window around a fixation point on a display. After a $400 \mathrm{~ms}$ fixation period, the stimulus was presented for $600 \mathrm{~ms}$, and if fixation had been maintained, a drop of juice was given as a reward. Stimuli were dichoptically presented using ferroelectric liquid crystal shutters (Displaytech) operating at a frequency of $60 \mathrm{~Hz}$ each and synchronized with the vertical retrace of the display monitor (20 inch monitor equipped with ultra-fast $\mathrm{P} 46$ phosphor; Vision Research Graphics) at a frequency of $120 \mathrm{~Hz}$ and a viewing distance of $86 \mathrm{~cm}$. No cross talk was measured between the images presented to the two eyes. Horizontal and vertical eye movements were recorded using an infrared-sensitive camera system sampling at $500 \mathrm{~Hz}$ (EyeLink II; SR Research).

During passive fixation, we searched for responsive neurons while disparity-defined 3D shapes, consisting of a combination of a twodimensional contour and a depth profile, were presented at the fixation point for $600 \mathrm{~ms}$. The stimulus set was identical to those used in previous experiments in AIP (Srivastava et al., 2009; Joly et al., 2009) and consisted of 32 3D shapes portraying curved surfaces, in which four disparity profiles were imposed over eight 2D contours (Fig. $2 A$ ) filled with a $50 \%$ density random dot pattern (stimulus size, $5.5^{\circ}$ ). Consistent with previous studies (Janssen et al., 2000b; Srivastava et al., 2009), we will refer to the combination of a $2 \mathrm{D}$ contour and a depth profile as a " $3 \mathrm{D}$ shape." On the basis of the responses in this test, the optimal 2D contour and two depth profiles (one of which the neuron responded strongly to, plus a less effective depth profile) were selected and further tested in a disparity test. Stimuli with opposite curvatures were created by interchanging the monocular images between the eyes (concave surfaces become convex and vice versa). Thus we created pairs of $3 \mathrm{D}$ shapes that used the same monocular images but contained opposite depth profiles. In the disparity test, we presented two pairs of $3 \mathrm{D}$ shapes together with monocular presentations of the same stimuli. Selectivity for disparity was assessed by comparing the responses to the two members of a pair of 3D shapes relative to the responses to monocular presentations of the same stimuli. A neuron was considered to be dispar- 
A

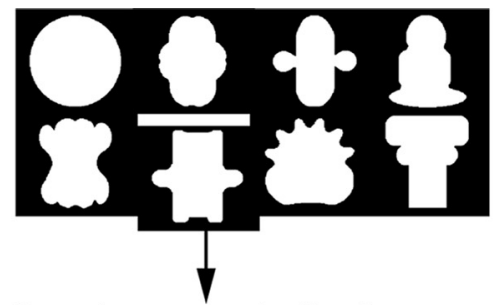

Gaussian
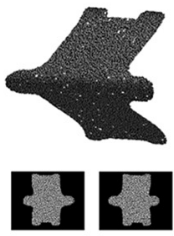

Sine
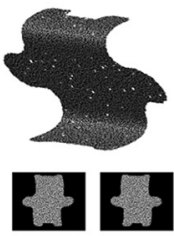

$\mathbf{B}$

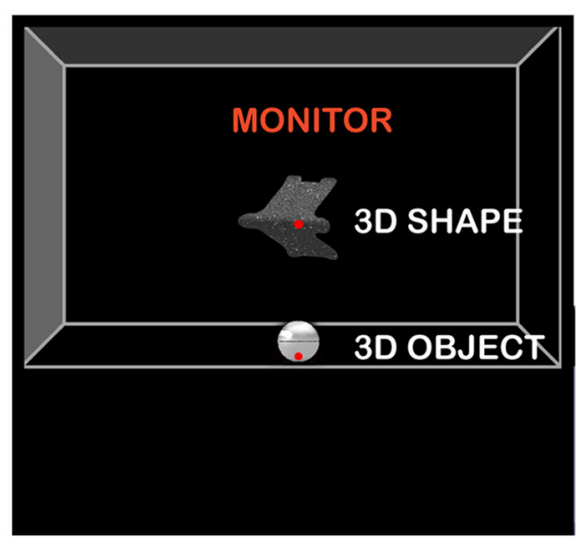

C

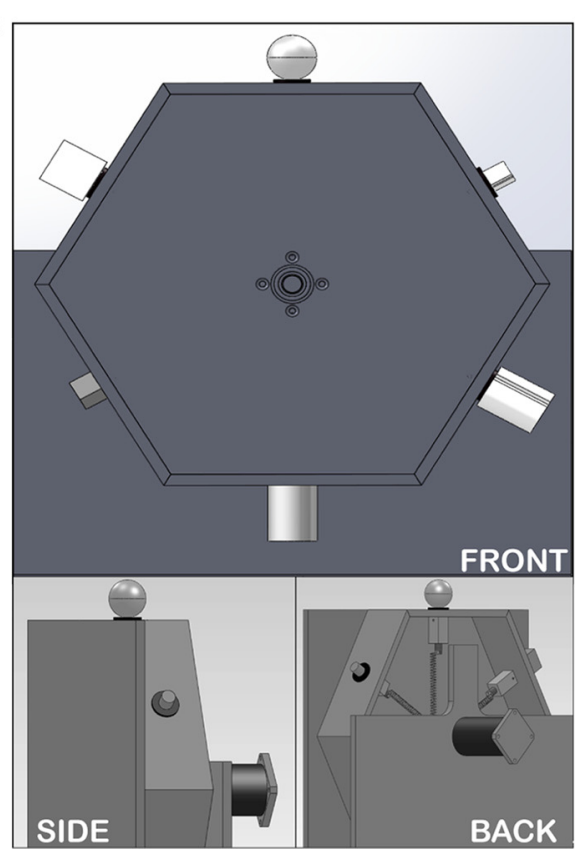

Figure 2. Stimuli and tasks. $\boldsymbol{A}$, The eight two-dimensional contours used to generate the 3D stimuli (top). Monocular images presented to the left eye and right eye and schematic illustrations ity selective if, for at least one of the 3D-shaped pairs, the response difference between the two members of that pair was significant $(t$ test, $p<$ 0.025 ) and at least three times greater than the difference between the monocular responses. Furthermore, we assessed the selectivity for higher-order disparity, i.e., selectivity for spatial variations of disparity, with a "position-in-depth test" (for details, see Janssen et al., 2000b) in which concave and convex $3 \mathrm{D}$ stimuli were presented at five positions in depth, ranging from $-0.50^{\circ}$ (near) to $+0.50^{\circ}$ (far) in steps of $0.25^{\circ}$. A neuron was considered to be responsive to the spatial variation of disparity (i.e., higher-order disparity selective or 3D-shape selective) if the response to the nonpreferred depth profile never significantly exceeded the response to the preferred depth profile at any of the positions-indepth tested. Although neurons that preserved their selectivities across positions in depth could be responsive to linear (first-order disparity) or curved (second-order disparity) surfaces, we will use the term "3D-shape selective" to indicate all neurons that were coding more than merely position in depth (zero-order disparity).

Grasping test. If a neuron was judged to be higher-order disparity selective, it was further tested in a visually guided grasping task. Seated in the dark, the monkey had to place its right hand in a resting position for $500 \mathrm{~ms}$, after which an LED was illuminated at the bottom of the object (Fig. $2 B$ ). Fixation on the LED (keeping the gaze inside a $2.5^{\circ}$ fixation window throughout the trial until the object was lifted) for $500 \mathrm{~ms}$ was followed by the illumination of a 3D object. Six different objects (a small cylinder and a small cube, both $15 \times 15 \times 15 \mathrm{~mm}$; a large cylinder, $35 \times$ $35 \times 35 \mathrm{~mm}$; a sphere, diameter $35 \mathrm{~mm}$; a large cube, $35 \times 35 \times 35 \mathrm{~mm}$; a cylinder with groove, cylinder $35 \times 35 \times 35 \mathrm{~mm}$; groove dimensions, $35 \times 7 \times 5 \mathrm{~mm}$ ) were pseudorandomly presented on a custom-built, vertically rotating carousel at a viewing distance of $28 \mathrm{~cm}$ (Fig. $2 \mathrm{~B}$ ). No attempt was made to create similarities between the objects in the grasping task and the disparity-defined 3D shapes shown in the previous task. In a given trial, only a single object was visible to the monkey. We tested F5a neurons with one, two, or six different objects, depending on the stability of the recording signal. After a variable period of 500 to $1000 \mathrm{~ms}$ of object fixation, an auditory GO cue instructed the monkey to reach, grasp, and lift the object. After a correctly completed trial, a juice reward was given. To isolate motor-related grasping activity, we also tested a subset of the neurons during grasping in the dark, where the identical task was performed in complete darkness (no LED and no object illumination), and a single object was used. Both monkeys were trained to perform right-handed grasping; all recordings were performed in the left hemisphere.

The resting position and the lifting phase were monitored by fiberoptic cables. Eye movements were recorded using an infrared-sensitive camera system (EyeLink II; SR Research). Eye position signals, neural activity, and photocell pulses were digitized and processed on a digital signal processor at $20 \mathrm{kHz}$ (C6000 series; Texas Instruments).

\section{Data analysis}

Disparity selectivity. Matlab (MathWorks) was used for data analysis. We first assessed the visual responsiveness of the neuron to the $3 \mathrm{D}$ stimuli in the disparity test ( $t$ test comparing the mean activity 50-450 ms after stimulus onset to the mean activity in the $400 \mathrm{~ms}$ preceding stimulus onset; $p<0.05$ ). For all subsequent analyses, net neuronal responses were calculated by subtracting the mean activity in the $400 \mathrm{~ms}$ interval preceding stimulus onset from the mean activity between 50 and $450 \mathrm{~ms}$ after stimulus onset.

The neuronal selectivity for disparity was tested using separate $t$ tests on the net responses to the two pairs of 3D shapes $(p<0.025)$. All

$\leftarrow$

of the perceived 3D structures (Gaussian, inclined, sine, half-period sine) are shown for one of the $2 D$ contours. $\boldsymbol{B}, A$ schematic overview of the experimental setup from the monkeys' viewpoint with the carousel presenting one of the six different $3 \mathrm{D}$ objects (bottom) and the monitor used to present the 3D shapes (top). Neurons were tested either during presentation of $3 \mathrm{D}$ shapes on the monitor (passive fixation) or during the grasping of an object. C, Front view (top), side view (bottom left), and back view (bottom right) of the carousel with the six different objects. Note that the carousel was invisible to the monkey. 
subsequent analyses were performed on the responses to the 3D-shape pair that evoked the strongest selectivity. To quantify the 3D-shape selectivity for a given pair of 3D stimuli, we calculated the stereo selectivity index (SSI), defined as SSI $=[$ (net response to the preferred 3D shape net response to the nonpreferred 3D shape)/net response to the preferred $3 \mathrm{D}$ shape], indicating the differential response normalized to the higher response for a given pair of $3 \mathrm{D}$ shapes. In the disparity test, we computed a stereo difference index (SDI), defined as SDI $=[($ difference in net response in the stereo condition - summed difference in net responses in the monocular conditions)/ (difference in net response in the stereo condition + summed difference in net responses in the monocular conditions)] (Janssen et al., 1999). As in our previous studies, the neuronal selectivity was considered to arise from binocular mechanisms (i.e., disparity selectivity) if the difference in responses to the two members of a pair of 3D shapes was significant $(t$ test, $p<0.025)$ and the SDI was $>0.5$, corresponding to a response difference in the stereo condition that was at least three times greater than the response difference in the monocular conditions of the same stimuli. We also quantified the degree of binocular summation by calculating a binocular summation index (BSI), which was defined as [ (net response to the preferred 3D shape in the stereo condition) - (sum of the net responses to the corresponding monocular presentations) $] /[$ (net response to the preferred 3D shape in the stereo condition $)+($ sum of the net responses to the corresponding monocular presentations)]. The response latency of the F5a population was calculated by identifying the first of three consecutive $10 \mathrm{~ms}$ time bins in which the population response to the preferred stimulus was significantly higher than that of the preceding time bin (Student's $t$ test, $p$ value $<0.05$, corrected for multiple comparisons). The latency of the neuronal population selectivity was likewise computed as the first of three consecutive $10 \mathrm{~ms}$ time bins in which the population response to the preferred 3D shape significantly exceeded the response to the nonpreferred 3D shape. For each individual neuron, we calculated the response latency based on a Poisson statistic (Janssen et al., 2008). The latency of the neural selectivity was calculated for each neuron using a sliding window analysis (window size, $50 \mathrm{~ms}$; step size, $10 \mathrm{~ms}$ ) as the first bin for which the response to the preferred $3 \mathrm{D}$ shape significantly exceeded the response to the nonpreferred 3D shape ( $t$ test, $p<0.05$ ).

To quantify neuronal preferences for particular $2 \mathrm{D}$ contours, we calculated the breadth of the selectivity of the responses in the search test (averaged for each 2D contour across the four depth profiles). The index $S_{\text {width }}$ was defined as $S_{\text {width }}=[(N-S U M / M A X) / N-1]$, in which $N$ is the number of stimuli in the test, SUM is the sum of all responses in the test, and MAX is the largest response in the test (Rainer et al., 1998). The $S_{\text {width }}$ index is 1 if the neuron responds to only a single $2 \mathrm{D}$ contour in the test, and 0 if the neuron responds equally to all 2D contours.

In the position-in-depth test, neurons were classified as responsive to the spatial variation of disparity if the response to the non-preferred shape did not significantly exceed any response to the preferred shape at any position in depth (Student's $t$ test evaluating the highest response to the nonpreferred shape compared to the lowest response of the preferred shape, $p<0.05$ ) (Janssen et al., 2000b).

Grasping test. Stable recordings of seven or more trials per condition were included for analysis. For the grasping task, net neuronal responses were calculated by subtracting the mean firing rate during the baseline epoch from the neuronal activity during the task. We calculated the activity elicited by object fixation as the mean firing rate, in the epoch between 50 and $450 \mathrm{~ms}$ after light onset, for the preferred object (defined as highest change from baseline), whereas movement-related activity was computed as the mean firing rate during the epoch between 300 and 700 $\mathrm{ms}$ after the GO cue during the reach-to-grasp phase. To verify that this analysis window was appropriate, we determined the average reaction time of monkey 1 (median, $423 \mathrm{~ms}$; first quartile, $329 \mathrm{~ms}$; third quartile, $549 \mathrm{~ms} ; N=4519$ trials) in a separate series of experiments. We classified neurons responding during visually guided grasping as "visual dominant" or "visuomotor" according to their responses in the dark (Sakata et al., 1995; Raos et al., 2006): visual-dominant neurons did not show a significant increase in neuronal activity during grasping in the dark ( $t$ test comparing the activity during the movement epoch to the baseline activity), whereas visuomotor neurons showed a significant increase in activ- ity during the movement epoch in the dark (exceeding five spikes per second to exclude neurons with weak but significant responses in the dark). Note that our criterion for visual-dominant activity did not require significant object-selective responses during object fixation, since we tested only a subset of the neurons during passive object fixation.

For the multiunit recordings during the grasping task, we performed offline spike sorting on the stored raw signal (sampling frequency 20 $\mathrm{kHz}$ ) using Offline Sorter (OFS; Plexon). Template matching of the action potential waveforms was performed using principal component analysis (PCA; valley-seeking algorithm). Single units were identified when the spikes formed a distinct cluster in a 2D solution of the PCA (i.e., a distinct spike waveform) and the number of interspike intervals of $1 \mathrm{~ms}$ or less was $<1 \%$. If either of these two criteria was not satisfied, the recording site was classified as multiunit.

\section{Results}

Our electrode penetrations first traversed the anterior bank of the inferior arcuate sulcus (area 45B) and then the sulcus, and finally entered the posterior bank of the inferior arcuate sulcus (Fig. 1). The pattern of transitions between gray matter and silent zones (the sulcus), combined with the depth of the recordings and the anatomical MRI allowed us to determine that our recordings were made in the posterior bank of the sulcus, which corresponds to the F5a region (Belmalih et al., 2009). Microstimulation in this region using currents up to $150 \mu \mathrm{A}$ did not evoke orofacial responses, suggesting that our recording area was not located in area 44 (Petrides et al., 2005). We recorded from 391 F5a neurons (205 in monkey 1 and 186 in monkey 2). A total of 260 neurons $(66 \%, 142$ in M1 and 118 in M2) showed significant responses to at least one of the 3D surfaces $(p<0.05, t$ test corrected for multiple comparisons) in the disparity test (visually responsive neurons).

\section{Responses of F5a neurons to disparity-defined curved surfaces}

Disparity test

The example neuron in Figure $3 \mathrm{~A}$ responded strongly (averaging 19 spikes/s in the epoch 50-450 ms after stimulus onset) to a disparity-defined convex 3D surface presented on a monitor. However, a concave surface composed of the same monocular images elicited much weaker responses (averaging only 3 spikes/s; $t$ test, $p<0.001$; SSI, 0.83 ). Monocular presentations of the convex and concave surfaces also evoked weak responses that did not differ between the two types of surfaces. Therefore, the significant selectivity observed in the stereo condition could not be explained by the monocular responses (SDI, 1.8), indicating disparity selectivity in this F5a example neuron. No significant differences in eye positions were detected between the convex and the concave surface presentations ( $t$ test on the mean eye position differences), and the average deviation of the eye positions (Fig. $3 A$ ) compared to baseline $\left(<0.05^{\circ}\right)$ was negligible compared to the disparity amplitude in the stimulus $\left(0.65^{\circ}\right)$. Note that this F5a neuron started signaling the difference between the depth profiles within $120 \mathrm{~ms}$ after stimulus onset (sliding window analysis with window size, $50 \mathrm{~ms}$; step size, $10 \mathrm{~ms}$; $t$ test on the differences in response to convex and concave surface, $p<0.05)$.

Significant differences between responses to convex and concave surfaces that could not be explained by the monocular responses (SDI, $>0.5$ ) were not uncommon in the F5a region that we recorded in: a total of 178 of 260 visually responsive neurons (68\%; 98/142 in M1 and 80/118 in M2) were disparity selective, and the proportions were nearly identical in the two monkeys $(69 \%$ and $68 \%)$. A small fraction of the neurons (7\%) showed an 
SDI of $<0.5$ and were excluded from further analysis. The median SDI in our population of 178 disparity-selective F5a neurons (1.1 for M1 and 1.3 for M2) was highly similar to data previously reported for AIP (median SDI AIP $_{1}$ 1.3) (Srivastava et al., 2009). [The sum of the monocular responses of the preferred $3 \mathrm{D}$ shape did not significantly differ from those of the nonpreferred 3D shape ( $t$ test, $p>0.5)$ ]. To quantify the degree of disparity selectivity, we calculated an SSI for the average net responses to the pair of $3 \mathrm{D}$ shapes showing the strongest selectivity (Fig. 3C). The median SSI was 0.71 ( 0.71 in M1 and 0.76 in $\mathrm{M} 2$ ), corresponding to a response to the preferred 3D shape 3.4 times greater than that to the other member of that pair. The distribution of the SSI (Fig. 3C) shows that neurons with weak disparity selectivities (SSI, <0.5) were relatively rare in F5a. Vergence eye movements in the disparity test were generally small $\left(<0.1^{\circ}\right)$ and occurred after neuronal selectivity had emerged (typically $\sim 200 \mathrm{~ms}$ after stimulus onset), and could therefore not account for the observed response differences. Thus, as predicted by the fMRI results reported by Joly et al. (2009), a sizeable proportion of F5a neurons are selective for binocular disparity.

Figure $3 B$ illustrates the time course of neuronal selectivity by plotting the average response to the preferred and nonpreferred 3D shapes of all disparity-selective F5a neurons for each of the two monkeys. Most neurons showed brisk responses to the $3 \mathrm{D}$ stimuli presented on the monitor: the time between the onset of the response and the maximum of the response [i.e., the highest bin in the peristimulus-time histogram (PSTH)] averaged only $60 \mathrm{~ms}$ (40 ms for M1 and $80 \mathrm{~ms}$ for M2). Furthermore, in both monkeys, the responses to our stereograms were robust and emerged shortly after stimulus onset. Notice the more tonic visual population response in M2 compared to M1. As early as $55 \mathrm{~ms}$ after stimulus onset in M1 and 65 ms after stimulus onset in M2, the population response begins to differ significantly from the baseline level (first of three consecutive $10 \mathrm{~ms}$ time bins significantly higher than the preceding time bin; $t$ test, $p<0.05$, corrected for multiple comparisons). Likewise, the neuronal selectivity became significant shortly after the onset of the response: as early as $75 \mathrm{~ms}$ after stimulus onset for M1 and 85 ms after stimulus onset for M2 (first of three consecutive $10 \mathrm{~ms}$ bins for which the population response to the preferred was significantly larger than the response to the nonpreferred depth profile; $t$ test, $p<0.05)$. We also analyzed the response latencies of the individual neurons

A

B

C
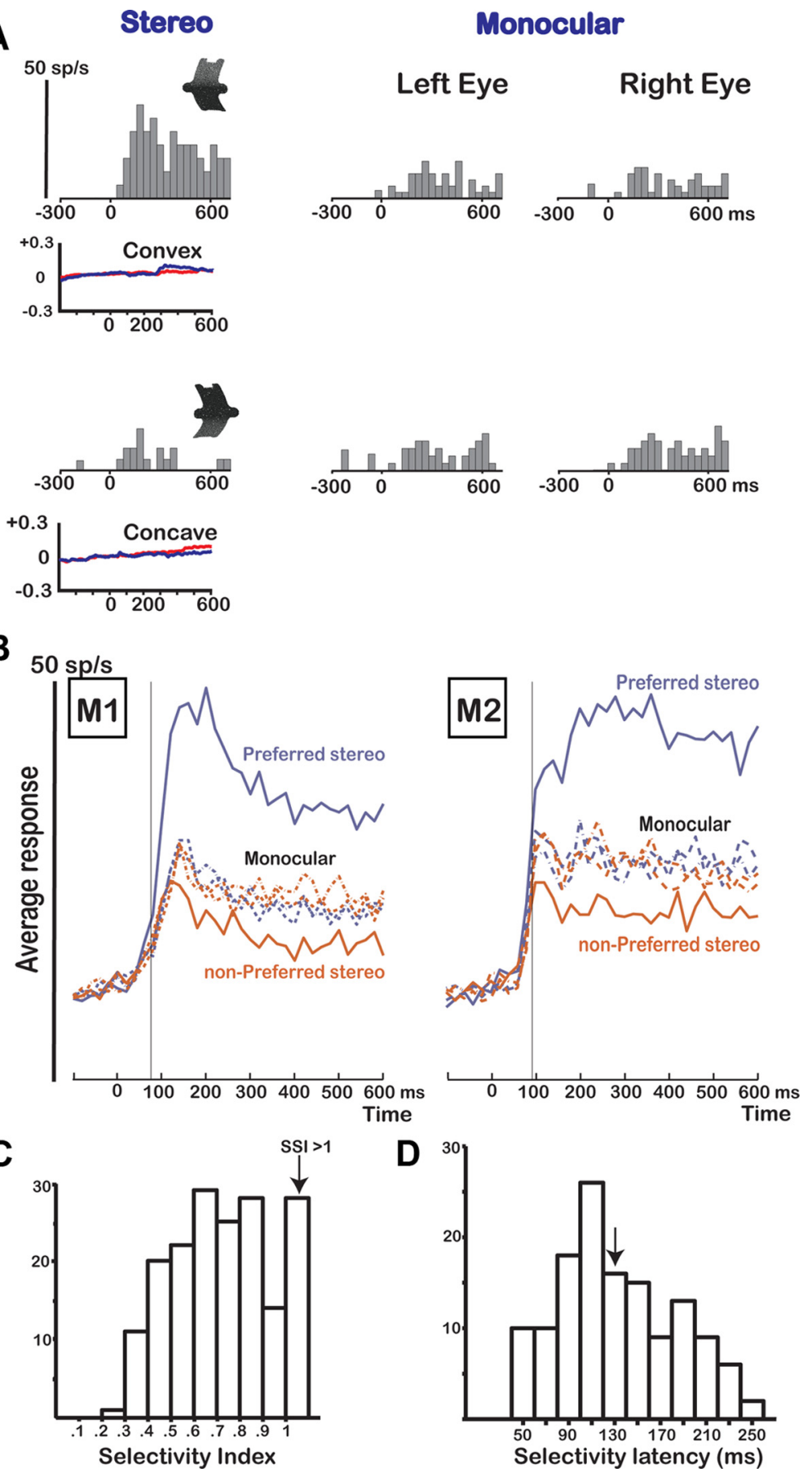

D

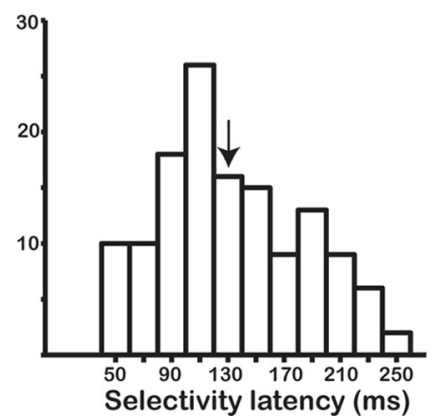

Figure 3. Disparity test. $\boldsymbol{A}$, Responses of an example neuron in area F5a showing selectivity in the disparity test. The icons illustrate the depth profile of the stimuli: convex (top row) and concave (bottom row). The neuron was tested with 3D shapes (stereo) and monocular presentations of the same stimuli (monocular left eye and right eye). The vertical calibration bar indicates 50 spikes per second (bin width, $40 \mathrm{~ms}$ ). Average horizontal eye position traces during presentation of the convex and concave shapes are plotted below the PSTHs for the left (red) and right (blue) eyes. $\boldsymbol{B}$, Population response of all disparity-selective neurons $(N=178)$ in $\mathrm{M} 1(N=98)$ and $\mathrm{M} 2(N=80)$. The average responses to the preferred (blue) and the nonpreferred 3D shapes (red) are plotted as a function of time after stimulus onset $(0)$. The thin vertical line indicates the latency of selectivity of the population response. The dashed lines represent the responses to the corresponding monocular stimuli. C, Histogram of SSI for all disparityselective F5a neurons $(N=178)$. The last bar includes all cells that had an SSI of 1 or higher. $\boldsymbol{D}$, Histogram of the selectivity latencies for all disparity-selective F5a neurons. The arrow indicates the median selectivity latency (130 ms). using a Poisson statistic (Srivastava et al., 2009; Theys et al., 2012). In this analysis the baseline activity is used to calculate the mean and SD of the spontaneous activity (which is assumed to follow a Poisson distribution), and the latency is calculated as the first of three consecutive time bins after stimulus onset where the 
activity significantly exceeds the level of spontaneous activity (Maunsell and Gibson, 1992; Janssen et al., 2008). The 10th percentile of response latencies was as little as $70 \mathrm{~ms}$ after stimulus onset in both monkeys, whereas the median response latency equaled $120 \mathrm{~ms}$ (110 ms for M1 and $120 \mathrm{~ms}$ for M2). To determine the selectivity latencies of individual F5a neurons, we determined at which point in time the response to the preferred $3 \mathrm{D}$ shape started to differ significantly $(t$ test, $p<0.05$ ) from the response to the nonpreferred $3 \mathrm{D}$ shape using a sliding window analysis (window size, $50 \mathrm{~ms}$; step size, $10 \mathrm{~ms}$ ). The median selectivity latency equaled $130 \mathrm{~ms}$, and the 10th percentile was 70 $\mathrm{ms}$, similar to the population selectivity latency. Figure $3 \mathrm{D}$ shows a histogram of the selectivity latencies in our population of F5a neurons. Thus, neurons in area F5a can signal disparity differences between curved surfaces at remarkably short latencies.

The example neuron in Figure $3 A$ also demonstrates that F5a neurons could show binocular summation, since the response to the preferred 3D shape was larger than the sum of the monocular responses (BSI, 0.34 for this neuron). However, the response to the preferred binocular stimulus was on average not larger than the sum of the monocular responses (median BSI, -0.03 for M1, -0.04 for M2).

A small fraction of the F5a neurons tested (14 of 391; 3.6\%) showed selectivity between the two pairs of 3D shapes (e.g., between the concave-convex pair and the inclined pair; Fig. 2A) but not between the two members of each pair (e.g., between the concave and the convex 3D shape). Because the two pairs in the disparity test used different monocular images, selectivity for the monocular images may have contributed to the observed response differences; therefore, these neurons were excluded from further analysis. Visually responsive F5a neurons that did not show any selectivity in the disparity test $(N=82,32 \%)$ were also not tested further. However, most responsive neurons in the search test showed a preference for a particular $2 \mathrm{D}$ contour in the search test. For the subset of neurons for which we had collected sufficient data in the search test $(N=99)$, we averaged the responses for each 2D contour across the four depth profiles and calculated the width of the selectivity $\left(S_{\text {width }}\right)$ (see Materials and Methods). The median $S_{\text {width }}$ equaled 0.56 ( $N=99$ neurons), which indicates that the neurons responded to an average of four of eight contours, similar to AIP neurons (median $S_{\text {width }}, 0.63$ ) (Srivastava et al., 2009). In the ranked responses averaged across neurons, half of the $2 \mathrm{D}$ contours elicited $<50 \%$ of the maximum response, and the worst contour in the test evoked no response on average. Thus F5a neurons also showed a preference for the 2D contour of our stimuli, which was similar to the contour selectivity previously reported for AIP neurons (Srivastava et al., 2009).

\section{Position-in-depth test}

The neuronal selectivity for convex versus concave surfaces could arise from a selectivity for between-stimuli differences in local disparities (e.g., the near disparities present in the center of the convex surface but not in the concave surface) rather than from a selectivity for the variation of the disparity along the vertical axis of the shape. Therefore we tested F5a neurons that showed selectivity for the two members of a pair of $3 \mathrm{D}$ shapes with curved surfaces at five positions in depth, with a mean disparity varying from +0.50 to $-0.50^{\circ}$. The example neuron in Figure $4 A$ (the same as in Fig. $3 A$ ) preserved its preference for the convex surface at every position in depth $(t$ test, $p<0.01$ ), indicating that the neuron was responding to the spatial variation of disparity along the vertical axis rather than to local disparities in the stimulus. In line with previous studies (Janssen et al., 2000b; Srivastava et al.,
2009; Theys et al., 2012), neurons that never responded significantly more strongly to the nonpreferred depth profile than to the preferred depth profile at any of the positions in depth tested (like the one in Fig. $4 A$ ), were considered higher-order disparityselective neurons.

Overall, 131 of 171 neurons tested (77\%) were higher-order neurons (62 in M1 and 69 in M2). Of the remaining 40 neurons, 18 neurons were lost during the transition from the disparity test to the position-in-depth test (as evidenced by the lack of selectivity in the position-in-depth test at the middle position, which was the same position as in the disparity test), whereas 22 neurons (13\%) significantly reversed their 3D shape preference at one or more positions (we termed these neurons zero-order neurons). Most higher-order F5a neurons ( 82 of $131 ; 63 \%$ ) preserved their selectivities for at least three of five positions tested $(p<0.10$ in view of the relatively small number of trials), but the proportion of neurons that showed significant selectivity at all five positions was small (12\%). Furthermore, 50\% of the neurons (66 of 131) showed a significant main effect of position, or a significant interaction between 3D shape and position (two-way ANOVA, $p<$ 0.05 ). Figure $4 B$ plots the average responses to the preferred and nonpreferred depth profiles for our population of higher-order neurons at each position in depth. Although the mean disparity of the stimulus exerted a strong influence in many F5a neurons, as a population the selectivity was clearly preserved at every position in depth ( $t$ test, $p<0.001$ at every position).

Vergence eye movements inside the $1^{\circ}$ fixation window could potentially influence the results of the position-in-depth test by reducing the mean disparity of the stimulus if our monkeys were to converge or diverge until the center disparity of the stimulus becomes zero. Therefore, we plotted the average positions of both eyes for the extreme near and far positions in depth $(-0.5$ and $+0.5^{\circ}$ mean disparity) separately for convex and concave surfaces (Fig. 4C). The most extreme stimulus positions (particularly the far positions) evoked small vergence eye movements, but the magnitude of this vergence response (maximum, $0.1^{\circ}$ in $\mathrm{M} 1$; $0.15^{\circ}$ in $\mathrm{M} 2$ ) was much smaller than the range of disparities in the test $\left(1^{\circ}\right)$. Furthermore, the vergence response emerged at least $200 \mathrm{~ms}$ after stimulus onset $(t$ test on the difference between the left and the right eye position, $p<0.05$; bin width $1 \mathrm{~ms}$ ), at a time when virtually all F5a neurons were already selective for the depth profile of the stimulus. Therefore, eye movements cannot explain the observed selectivity for convex and concave surfaces in area F5a.

Overall, as predicted by the fMRI study of Joly et al. (2009), a large proportion of premotor area F5a neurons were higherorder disparity selective. These F5a neurons showed brisk and highly selective responses to random-dot stereograms presented in the display, and the latency of the selectivity was remarkably short.

\section{Responses in neighboring periarcuate regions}

We also recorded from 20 responsive single neurons in the more posterior F5 regions ( $\mathrm{F} 5 \mathrm{p} / \mathrm{c})$. We found no disparity-selective neurons in this portion of F5, although visual responses were present, as shown in the average population response in Figure $5 A(N=20)$. Furthermore, the temporal pattern of the responses differed markedly between the F5p/c recording sites and F5a: instead of the brisk visual responses in area F5a, the neurons in these more posterior positions invariably showed sluggish responses that evolved over several hundreds of milliseconds.

In addition, we recorded the activity of 63 responsive [ $t$ test on the spike rate in the interval ( 50 to $450 \mathrm{~ms}$ ) after stimulus onset 

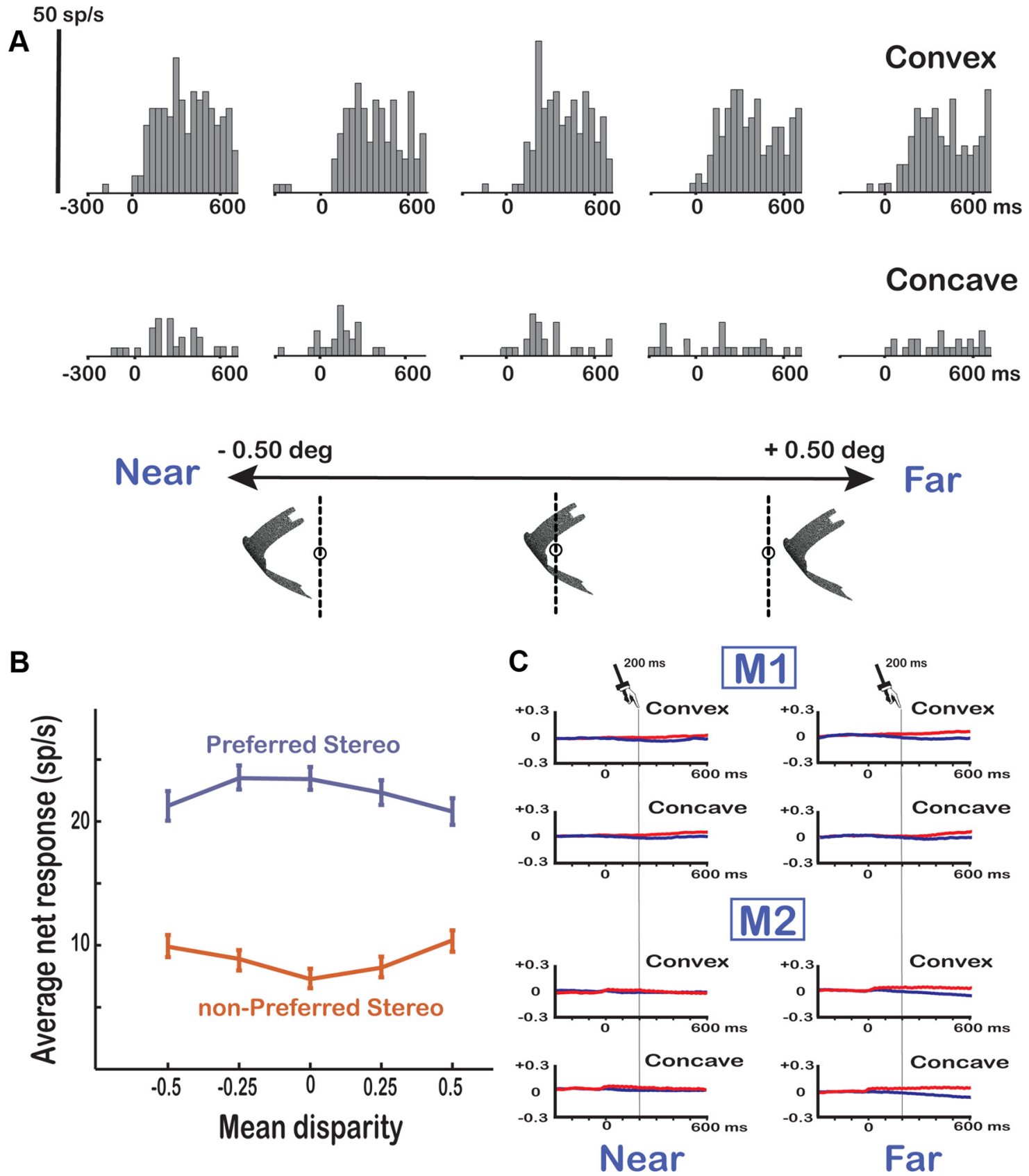

Figure 4. Position-in-depth test. $A$, Example neuron. A higher-order 3D shape selective neuron in area F5a (the same neuron as in Fig. 3A) showing robust responses to the preferred 3D shape at all positions in depth is shown. The vertical calibration bar indicates 50 spikes per second (bin width, $40 \mathrm{~ms}$ ). $\boldsymbol{B}$, Population response of higher-order selective $F 5$ a neurons ( $N=131$ ). The average net responses of all higher-order neurons to the preferred (blue) and the nonpreferred (red) $3 \mathrm{D}$ shape are plotted at the five different positions in depth. C, Eye position traces. Average horizontal eye position traces for the left (red) and right (blue) eyes are shown for the extreme near (left) and extreme far (right) positions in the position-in-depth test for the two monkeys (M1, M2) separately.

compared to baseline, $p<0.05$ ] single neurons in area $45 \mathrm{~B}$ on the anterior bank of the inferior arcuate sulcus. Thirty-four cells (54\%) showed significant selectivity for the two members of a pair of 3D shapes (e.g., concave versus convex) that could not be explained by the monocular responses and were therefore considered to be disparity selective. The average population response of these 34 neurons is shown in Figure 5B. The neuronal selectivity in 45B was robust (median SSI, 0.42) and emerged after $90 \mathrm{~ms}$ ( $t$ test on the population responses to preferred and nonpreferred 3D shape, 20 ms bins).

However, although a substantial proportion of $45 \mathrm{~B}$ neurons showed disparity selectivity, most of these 45B neurons did not preserve their selectivities across positions in depth. The large majority of $45 \mathrm{~B}$ neurons ( $82 \%$ ) showed a significant reversal in the $3 \mathrm{D}$-shape preference in the position-in-depth test, indicating selectivity for zero-order disparity but not for higher-order disparities. For each neuron, we plotted the selectivity index at the middle position (SIm) against the selectivity index at the worst position (SIw) (comparing the smallest response to the preferred $3 \mathrm{D}$ shape to the largest response to the nonpreferred 3D shape). For most neurons in area 45B (Fig. 5C), the SIw was negative, indicating a stronger response to the nonpreferred $3 \mathrm{D}$ shape. In contrast, the majority of disparity-selective neurons in area F5a $(N=131$ of $171 ; 77 \%)$ preserved their selectivities across positions in depth, indicating higher-order disparity selectivity. For comparison with area $45 \mathrm{~B}$, we plotted the SI at the middle posi- 

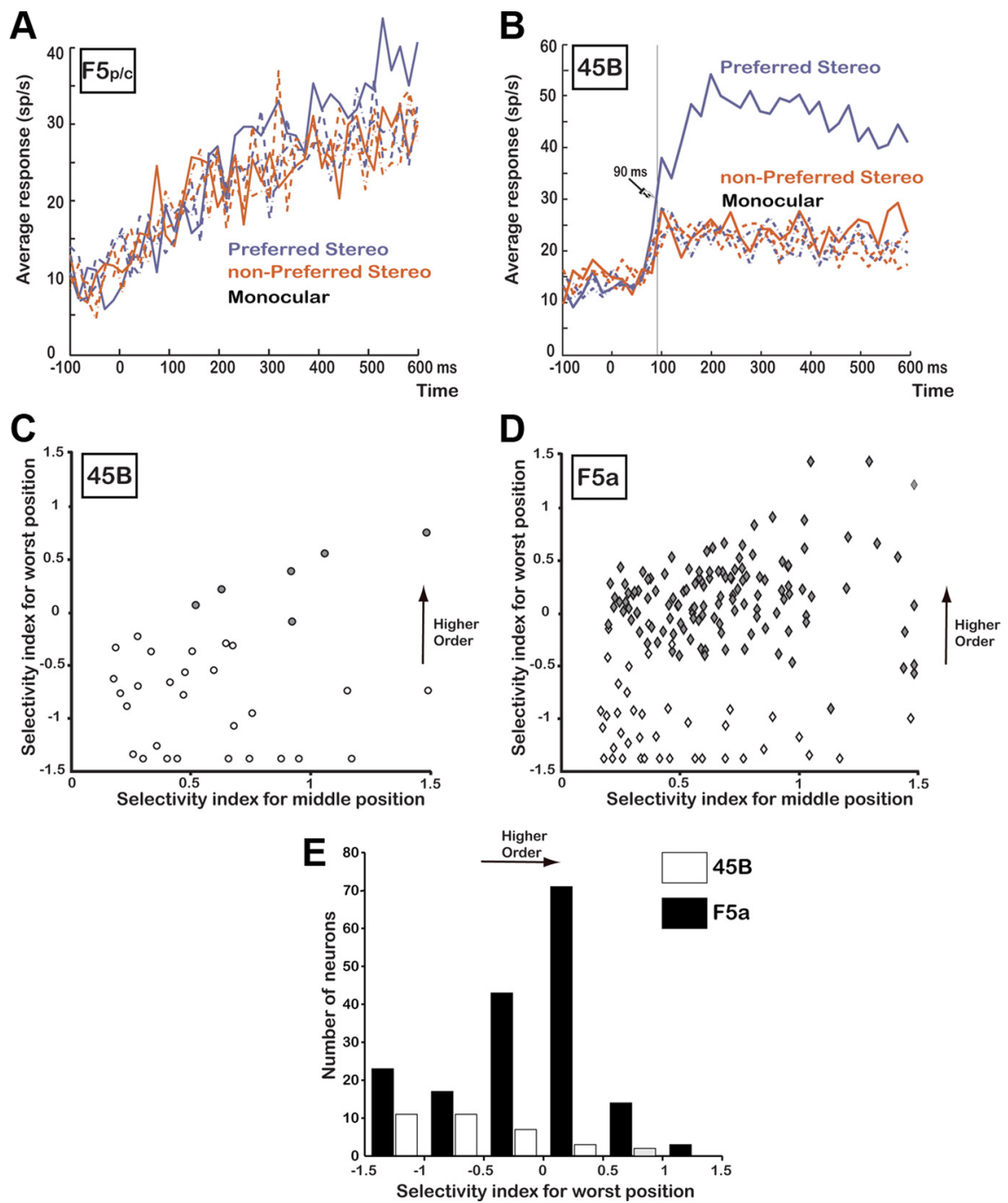

Figure 5. Responses in other periarcuate regions. $A$, Average response of all responsive neurons recorded in $\mathrm{F5p} / \mathrm{C}(N=20$; same conventions as in Figure 3C). $\boldsymbol{B}$, Average response of all disparity-selective neurons recorded in area $45 \mathrm{~B}(\mathrm{~N}=34$; same conventions as in Figure 3C). C, Selectivity index at the middle position plotted against the selectivity index at the worst position (i.e., the smallest response to the preferred 3D shape compared to the strongest response to the nonpreferred 3D shape) for all disparity-selective neurons in area 45B $(N=34)$. Open symbols, Zero-order neurons; filled symbols, higher-order neurons. $\boldsymbol{D}$, The same selectivity indices plotted for all disparity-selective neurons recorded in $\mathrm{F5a}(N=171)$. $\boldsymbol{E}$, Histogram of the selectivity index at the worst position for all disparity-selective neurons recorded in F5a ( $N=171$; filled bars) and area 45B ( $N=34$; open bars).

tion against the SI at the worst position for all F5a neurons that showed disparity selectivity (Fig. 5D). Figure $5 E$ shows a histogram of the SIw for the neurons recorded in F5a (filled bars) and the neurons recorded in area 45B (open bars). Since no disparity selectivity was observed in $\mathrm{F} 5 \mathrm{p} / \mathrm{c}$, we did not perform the position-in-depth test in this area. Both F5a and area 45B contained zero-order neurons, but higher-order neurons were almost exclusively recorded in F5a. Thus, although visual responses could be recorded over an extended range in and around the arcuate sulcus, higher-order disparity-selective neurons were concentrated in area F5a, in line with the results of Joly et al. (2009).

\section{Grasping activity in area F5a}

The region of F5a we recorded from contained many neurons encoding the depth profile of curved surfaces during passive fixation of random-dot stereograms presented on a display. We wondered to what extent these higher-order disparity-selec- tive F5a neurons also exhibited the more established properties of ventral premotor neurons, i.e., grasping-related activity (Murata et al., 1997; Raos et al., 2006). To answer this question, we tested a subpopulation of higher-order disparity-selective F5a neurons in a delayed grasping task both in the light and in the dark.

\section{Visual-dominant and visuomotor activity in F5a: example sites}

The F5a example neuron in Figure 6 was first tested during passive fixation with concave and convex random-dot stereograms presented on the display. The neuron fired strongly and selectively to a convex depth profile but much less strongly to a concave depth profile (Fig. $6 A$ ), a selectivity that could not be accounted for by the pattern of the monocular responses (data not shown). Furthermore, the neuron preserved its selectivity across positions in depth, with significant $3 \mathrm{D}$ selectivity at four of five positions ( $t$ test, $p<0.05$; data not shown). After having completed the position-indepth test, we recorded the same neuron during grasping of the large cube. Figure $6 B$ shows the activity of the same neuron during grasping, aligned with the onset of illumination (panel), on the GO signal (middle), and on the lifting of the object (right) in the light (top) and in the dark (bottom). (Time 0 in the left panel in the bottom row corresponds to the time when the light would have switched on during grasping in the light.) The example neuron showed a strong response $(p<0.001$ stimulus epoch compared to baseline epoch) after the onset of the illumination of the object (Fig. $6 \mathrm{~B}$, top, Light), which remained present until the monkey had reached for, grasped, and lifted the object.

During grasping in the dark, however, the activity of this example neuron was strongly reduced (Fig. $6 \mathrm{~B}$, bottom row): although a slight elevation of the activity above baseline could be detected $(t$ test on the average response during grasping compared to baseline, $p<0.05$ ), the activity during the movement phase in the dark was much weaker than in the light (on average, less than five spikes per second). We labeled neurons for which the neuronal activity during grasping in the dark was no higher than baseline or neurons where the net neuronal activity was less than five spikes per second as visual dominant. (A statistically significant response in the dark compared to baseline would have included neurons with very weak responses in the dark, such as the example neuron in Figure $6 \mathrm{~B}$.) We also tested the same neuron during passive fixation of two objects (a large cube and a small cube) and observed significant object selectivity during fixation ( $p<0.01, t$ test; data not shown). To illustrate that the data presented in Figure $6 B$ were derived from a single neuron, we plotted a short section of the raw signal trace during visually guided grasping in Figure $6 \mathrm{C}$. Therefore, despite considerable 
differences in visual features between the random-dot stimuli and the objects, higher-order disparity-selective F5a neurons also responded strongly to realworld objects during visually guided grasping. In contrast to previous studies in F5 (Murata et al., 1997; Raos et al., 2006), we observed visual-dominant neurons that fired strongly during grasping in the light but not during grasping in the dark.

We also recorded multiunit activity (MUA) during the stereo tests, grasping in the light, and grasping in the dark, and observed markedly different response properties, in particular during grasping in the dark. The example MUA site in Figure 7 was clearly selective for the depth profile of curved surfaces (Fig. 7A), which was preserved across positions in depth (data not shown). Furthermore, the MUA responded strongly during grasping in the light, during the object fixation epoch, and throughout the delay period, as well as during the movement epoch (Fig. $7 B$, top). In contrast to the single-unit example in Figure 6, the activity in the dark during the movement epoch was almost as strong as in the light, indicating visuomotor activity (Fig. $7 B$, bottom). We recorded several units simultaneously during the grasping task (both in the light and in the dark), as can be seen in the raw recording signal containing several spike waveforms (Fig. 7C). MUA has to be interpreted with caution, however, because different units may exhibit different selectivities, i.e., the stereo-selective unit(s) could have differed from the visuomotor unit(s). But at the very least, the MUA recording site shown in Figure 7 demonstrates two new findings. First, not all F5a neurons are visual dominant, since one or more units in this MUA recording site were also active in the dark. Second, Figure 7 also shows that F5a neurons encoding the depth profile of surfaces were either identical to, or located in close proximity to (a few hundred micrometers from), neurons that were active during object grasping in complete darkness, i.e., visuomotor neurons. For the example shown in Figure 7, the spike-sorting analysis performed on the stored raw signal revealed that the activity in the dark was almost exclusively caused by the small spikes, while the two large spikes were visual dominant (the largest being $3 \mathrm{D}$-shape selective, indicated by the asterisks in Fig. 7C).

\section{Population analysis of grasping activity in F5a}

In total, we recorded activity in 98 higher-order disparityselective sites [46 single-unit activity (SUA), 52 MUA] in F5a during visually guided grasping. The great majority of 3D-shapeselective sites ( 72 of $98,73 \%$ ) also responded significantly during grasping in the light. Figure 8 shows the net response (mean activity after subtraction of the baseline activity before the onset of the light) during the object fixation epoch (50-450 ms after light onset) plotted against the net response during the movement ep- och (300-700 ms after GO cue) for all 98 recording sites, during grasping in the light, and separately for SUA sites and MUA sites. Clearly, the responses we measured during grasping in the light were very robust and similar for SUA sites and MUA sites. Furthermore, Figure 8 also shows that the activity in these two trial epochs was highly correlated (correlation coefficient, $0.75 ; p<$ 0.001 ). Indeed almost none of the recording sites showed activity during the movement epoch but not during object fixation, as in strictly motor-dominant neurons (Raos et al., 2006). Note that the high proportion of grasping responses we observed is remarkable in view of the difference in viewing distance between the objects $(28 \mathrm{~cm})$ and the random-dot stereograms presented on the display $(86 \mathrm{~cm})$ (see Materials and Methods), and the difference in gaze direction (see Fig. 2B), suggesting that these factors may not be crucial for most F5a neurons.

To determine whether higher-order disparity-selective F5a neurons could also exhibit visuomotor activity (i.e., activity during visually guided grasping and during grasping in the dark), we tested the activity in the dark of 50 recording sites (24 SUA, 26 MUA) that had demonstrated selectivity for the depth profile of curved surfaces and grasping activity in the light. The great majority of SUA sites $(20$ of $24 ; 83 \%)$ were visual dominant (like the 
A

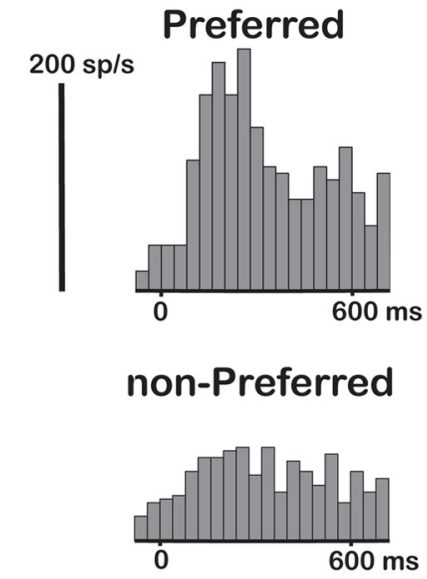

B
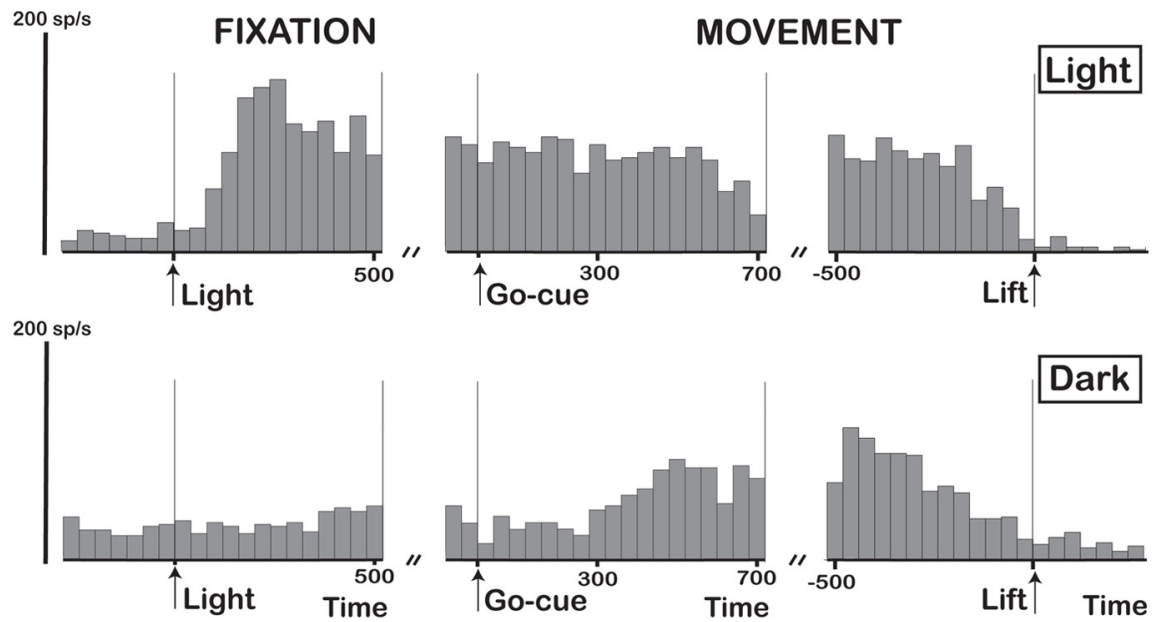

Figure 7. Grasping-related activity: MUA. A, PSTH of an example MUA recording site showing 3D shape selectivity. $\boldsymbol{B}$, Grasping task. A PSTH of the same disparity-selective 3D site tested during grasping is shown. Same conventions as in Figure 6B. C, Raw signal trace (top) and magnified trace (bottom) recorded during grasping in the light illustrating MUA. Asterisks indicate the disparity-selective spikes, and diamonds indicate the visuomotor spikes.

example neuron in Fig. 6), whereas the MUA sites frequently fired strongly in the dark ( 16 of $26 ; 62 \%)$. Although we occasionally encountered single units that were both $3 \mathrm{D}$-shape selective and active during grasping in the dark $(N=4)$, most of the activity in the dark ( 16 of $26 ; 62 \%$ ) was caused by small spikes in the MUA (possibly interneurons) (but see Vigneswaran et al., 2011) that were not selective for $3 D$ shape, as in the example in Figure 7. In general, single 3D-shape-selective F5a neurons were visual dominant but located in close proximity to visuomotor neurons.

Motor-dominant neurons in F5 respond during the movement epoch in both the light and the dark (Raos et al., 2006), but not during object fixation, whereas visuomotor neurons fire during object fixation and during grasping in the dark. In our data set of F5a neurons, a large majority of recording sites that were active during grasping in the dark (15 of 20; 75\%; 16 MUA, 4 SUA) responded during the first $300 \mathrm{~ms}$ of the object fixation epoch $(0-300 \mathrm{~ms}$ after light onset), and almost all of them responded in the first $500 \mathrm{~ms}$ of object fixation (19 of 20; 95\%), and can therefore be considered visuomotor. This does not imply that motordominant neurons do not exist in area F5a, since we observed motor-dominant activity (i.e., activity during the movement epoch in both the light and in the dark) in sites that were not 3D-shape selective, and sometimes even in the same electrode penetration within a few hundred microns of 3D-shape-selective recording sites (data not shown).

Figure $9 A$ shows the average activity of all 98 higher-order disparity-selective F5a recording sites (46 SUA, $52 \mathrm{MUA}$ ) during grasping in the light, aligned with light onset (left), GO cue, and object lift (right). Our population of F5a neurons fired strongly when the light came on, and continued to fire throughout the delay period until the object was lifted. The onset of the light above the objects (Fig. 9A, time 0 ) evoked less brisk responses than the onset of the 3D surfaces on the display (compare Fig. 3C). However, we searched for neurons during presentations of 3D surfaces and selected the best stimulus for each neuron, whereas the objects we selected for the grasping task may have been suboptimal for the neurons tested. Figure $9 B$ shows the average activity during grasping in the dark, independently for visual-dominant recording sites and visuomotor sites. In the dark, the activity in the visuomotor recording sites was very robust (Fig. 9B), while the larger fraction of visual-dominant neurons showed virtually no activity. Notice the strong rise of activity in visuomotor F5a neurons in anticipation of the GO cue (Fig. 9B, left), which is reminiscent of the climbing activity in LIP (lateral intraparietal area) neurons during saccade tasks (Janssen and Shadlen, 2005; Premereur et al., 2011).

\section{Object selectivity in F5a}

We recorded 59 F5a neurons tested with two or more different objects during visually guided grasping. We observed significant object selectivity during the object fixation epoch in $50 \%$ of $3 \mathrm{D}$-shape-selective neurons tested with more than one object $(t$ test, $p<0.05)$. The relatively low proportion of object selectivity was likely caused by the high degree of similarity between our objects, which almost exclusively consisted of convex depth profiles (Fig. 2), and the fact that we did not search for responsive neurons during grasping. Thirteen neurons were tested during passive fixation of the object in the absence of a grasping response, almost all of which (12 of 13) responded significantly to at least one of the objects. Conversely, the neuronal preference for a particular depth profile did not clearly match the geometry of the object to which the neurons responded: although all our objects were essentially convex, we observed a wide range of depth-profile preferences (i.e., selectivity for the random dot stereograms) in the population of 72 neurons that responded during grasping ( $42 \%$ of cells preferred a convex $3 \mathrm{D}$ shape, $26 \%$ preferred a concave $3 \mathrm{D}$ shape, and $32 \%$ preferred one of the inclined surfaces).

We did not test whether our 3D-shape-selective F5a neurons were also encoding the depth profile of the objects during visually guided grasping. It should be noted that assessing $3 \mathrm{D}$ visual selectivity with real-world objects is not trivial: monocular object presentation would remove disparity information, but would 
leave all monocular depth cues (perspective, shading, texture, etc.) intact, and presenting objects with identical contours but different depth profiles is very difficult given that the object contours usually follow the depth structure in real-world objects. The advantage of random-dot stereograms is that disparity is the only depth cue and that different depth profiles with identical 2D contours can be presented.

\section{Discussion}

Our study is the first functional characterization of F5a at the single-cell level. We found strong selectivity for the depth profile of curved surfaces (higher-order disparities) in a large proportion of F5a neurons, as predicted by a previous monkey fMRI study (Joly et al., 2009). The same neurons also responded to real-world objects during visually guided grasping. Neurons selective for higher-order disparities were generally not active in the dark but frequently colocalized with visuomotor neurons. Consistent with the known anatomical connectivity (Gerbella et al., 2011), our results indicate that F5a can be considered a pre-premotor area involved in object grasping with a specific role in the visual analysis of $3 \mathrm{D}$ object properties.

Our data provide critical evidence that $\mathrm{F} 5 \mathrm{a}$ is indeed distinct from the other F5 subdivisions in view of the concentration of 3D-shape-selective neurons and the presence of visual-dominant neurons in F5a. Moreover the presence of visualdominant neurons encoding 3D object information in F5a also provides a functional interpretation of the concept of pre-premotor cortex, as suggested by Gerbella et al. (2010): the pre-premotor F5a sector is clearly much more visual than the other F5 sectors, where previous studies have observed no visual-dominant neurons (Raos et al., 2006). Fluet et al. (2010), however, also described the presence of "sensory" (visual-dominant) neurons in F5. These authors reported a higher concentration of sensory orientation-tuned neurons in a dorsal and a ventral F5 region, which they related to the sites in F5 with strong AIP inputs described by Borra et al. (2008). It is conceivable that one of these F5 regions with sensory neurons described by Fluet et al. (2010) may have at least partially corresponded to F5a. Note that the so-called "canonical" neurons, which respond during object fixation and during object grasping, were recorded in F5p (Rizzolatti et al., 2002).

Several lines of evidence indicate that we recorded in F5a and not in the adjacent area 44 in the fundus of the inferior arcuate bank. First, the reconstruction of the recording sites based on the anatomical MRI indicated that our penetrations entered the posterior bank of the inferior arcuate sulcus (Fig. $1 B$ ). A comparison between the sagittal MR sections in Figure $1 A$ and Figure $4 A$ of Belmalih et al. (2009) clearly demonstrates that our recording positions were located posterior to area 44. Second, microstimulation in 3D-shape-selective F5 sites did not evoke orofacial (nor limb or eye movement) responses, in contrast to area 44 (Petrides et al., 2005), even with much stronger currents (up to $150 \mu \mathrm{A}$ ) than those used in the area 44 study $(15-60 \mu \mathrm{A})$. Third, the great majority of 3D-shape-selective neurons that we recorded from, were active during grasping in the are plotted separately.

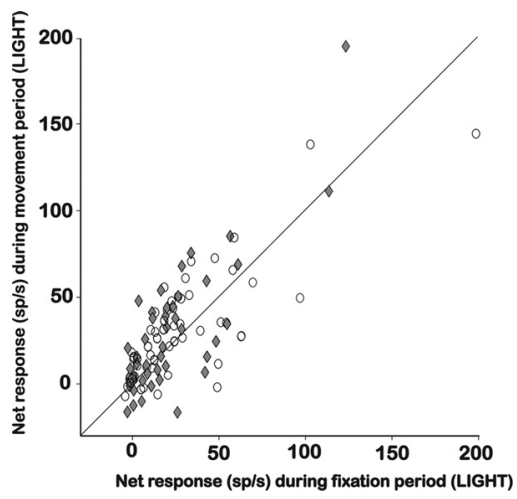

Figure 8. Grasping task in the light: population analysis. For all 3D-shape-selective F5a neurons tested during visually guided grasping $(N=98)$, the net neuronal activity (spikes per second) during the fixation period is plotted against the activity during the movement period for the SUA sites ( $N=46$, filled diamonds) and the MUA sites ( $N=52$, open circles).
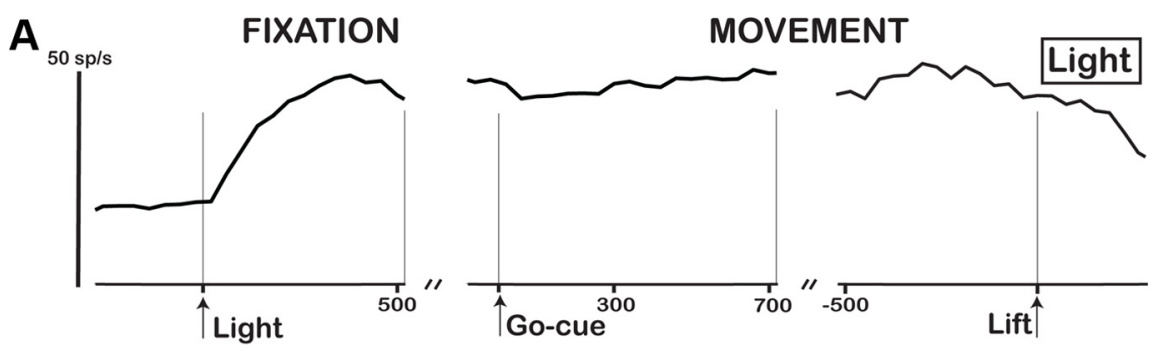

MOVEMENT
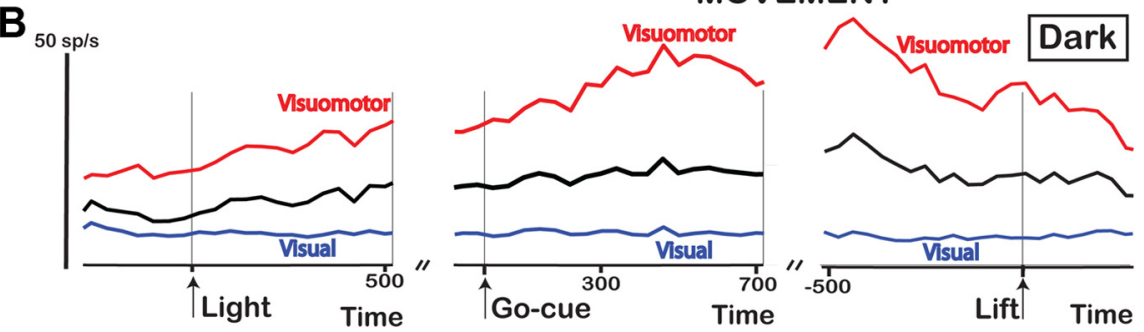

Figure 9. Grasping task: average population response. $\boldsymbol{A}$, The average response of all 3D-shape-selective F5a neurons (black $N=98$ ) tested during grasping in the light is plotted as a function of time after stimulus onset (left), at the time of $\mathrm{GO}$ cue (middle), and at the time of object lift (right). $\boldsymbol{B}$, The average response of all 3D-shape-selective sites ( $N=50$; black line) tested during grasping in the dark is plotted as a function of time after stimulus onset (left), time of $\mathrm{GO}$ cue (middle), and time of object lift (right). The average responses of all higher-order visual (blue line; $N=30$ ) and visuomotor (red line; $N=20$ ) recording sites

light, and nearby neurons were also active during grasping in the dark, consistent with the known properties of ventral premotor neurons.

The F5a region that was activated more strongly by curved surfaces than by flat surfaces at different positions in depth was confined to the depth of the posterior bank of the inferior arcuate sulcus (Joly et al., 2009), but F5a occupies the more anterior part of the posterior bank of this sulcus (Belmalih et al., 2009). We typically found 3D-shape-selective neurons in three to four recording positions targeting this region (an extent similar to area AIP) (Srivastava et al., 2009), but not in more lateral, anterior, or posterior recording positions. Therefore, the possibility exists that the region of F5a we recorded from is distinct from the rest of F5a. Alternatively, the limited stimulus set we used in the search test may have been responsible for the relatively restricted region in which we observed 3D-shape selectivity, which implies that with a larger and more diverse stimulus set, we may have found responses across a wider area. A similar phenomenon has been 
described for IT (Janssen et al., 2000a; Yamane et al., 2008). Overall, it is highly likely that the population of 3D-shape-selective F5a neurons we recorded represents a subset of a much larger population of F5a neurons with object responses during grasping.

The neuronal selectivity for disparity-defined curved surfaces in F5a was highly similar to that in area AIP in terms of the proportion of disparity-selective neurons (68\% in F5a compared to $83 \%$ in AIP) and the proportion of higher-order neurons ( $77 \%$ in F5a compared to $83 \%$ in AIP), and the strength of this selectivity appeared even stronger in F5a (SSI, 0.71) than in AIP (SSI, 0.64) (Srivastava et al., 2009). Furthermore, the population latency of the neuronal selectivity in F5a (75-85 ms) was slightly longer than that of AIP (50-70 ms) (Srivastava et al., 2009; Theys et al., 2012), but nevertheless shorter than in IT (100-120 ms) (Janssen et al., 1999; Verhoef et al., 2011). F5a neurons tended to preserve their selectivities across fewer positions in depth (median number of positions, 3 ) compared to AIP (median number of positions, 4), suggesting that mean disparity exerts a stronger influence in F5a than in AIP. Overall, the selectivities and latencies of F5a neurons are compatible with the hypothesis that AIP sends 3D-object information to F5a. Future studies will have to investigate whether the representation of 3D shape in F5a differs from the known representation of 3D shape in AIP.

Our description of higher-order disparity selectivity in ventral premotor cortex complements previous studies on IT (Janssen et al., 2000a) and AIP (Srivastava et al., 2009). IT neurons provide a very elaborate and categorical representation of $3 \mathrm{D}$ object features (Janssen et al., 2000b), the activity of 3D-shape-selective IT neurons correlates with perceptual choice during 3D-shape categorization (Verhoef et al., 2010), and microstimulation of 3Dshape-selective clusters of IT neurons predictably influences the perceptual choice of monkeys during 3D-shape categorization (Verhoef et al., 2012). AIP neurons, in contrast, encode 3D information in a faster, coarser, and more metric (as opposed to the categorical coding in IT) manner (Srivastava et al., 2009). The correlation between AIP activity and perceptual choice emerges late and after the decision has been formed when monkeys categorize 3D shape (Verhoef et al., 2011), and reversible inactivation of 3D-shape-selective AIP sites produces a grasping deficit but no deficit in 3D-shape categorization (Verhoef et al., 2009). Here, the preponderance of 3D-shape-selective F5a neurons that respond during object grasping also indicates a different role for higher-order disparity selective neurons, i.e., object analysis for the purpose of grasping.

F5a neurons encoding 3D object properties were generally not active during grasping in the dark and should therefore be considered visual dominant. Our MUA recordings indicate that 3Dshape-selective neurons were frequently embedded in clusters of visuomotor (but not motor-dominant) neurons. Therefore, ensembles of visual-dominant and visuomotor F5a neurons may constitute functional units in which visual 3D object information is transformed into a more motor-specific code, which is subsequently transmitted to motor-dominant neurons in F5a and in the other F5 sectors. Consistent with this proposal, Raos et al. (2006) reported that F5 visuomotor neurons represent objects in motoric terms, i.e., determined by the grip type used to grasp the object but not by the visual object features (like AIP neurons do). Presumably Raos et al. (2006) did not record in the depth of the posterior bank of the inferior arcuate sulcus and therefore did not find visual-dominant neurons. In any case, our data furnish a new view concerning the pathways involved in visuomotor transformations, with F5a occupying a pivotal role in the intricate process of visuomotor transformations. Visual, 3D object infor- mation could be sent from AIP to F5a visual-dominant neurons, where it could be transmitted to visuomotor neurons and subsequently sent to other F5 subregions (F5c and F5p) to drive primary motor cortex or spinal cord neurons. It remains to be determined to what extent this hypothesis is true.

We observed neuronal selectivity in F5a (3D-shape-selective, visual-dominant clusters of neurons, but also visuomotor and motor-dominant activity during grasping) similar to that seen in parietal area AIP (Murata et al., 2000; Srivastava et al., 2009). We also observed strong grasping activity in the majority of 3D-shape selective AIP sites (Theys, Pani, and Janssen, unpublished observations). At this point we can only speculate what the differences between AIP and F5a might be. It is remarkable that in our study, few F5a neurons showed motor-dominant activity (i.e., activity during the movement epoch but no activity during the object fixation epoch), while this type of activity is quite common in AIP and in the other F5 subregions (Murata et al., 1997, 2000; Raos et al., 2006). One intriguing possibility is that visual-dominant and visuomotor F5a neurons are more heavily involved in the transformation of a visual object representation (F5a) into a motor object representation (Raos et al., 2006), while motor-dominant activity in AIP could reflect a corollary discharge from feedback projections originating in ventral premotor cortex that can be used in online visual control during object grasping (Taira et al., 1990; Rizzolatti and Luppino, 2001).

\section{References}

Belmalih A, Borra E, Contini M, Gerbella M, Rozzi S, Luppino G (2009) Multimodal architectonic subdivision of the rostral part (area F5) of the macaque ventral premotor cortex. J Comp Neurol 512:183-217.

Borra E, Belmalih A, Calzavara R, Gerbella M, Murata A, Rozzi S, Luppino G (2008) Cortical connections of the macaque anterior intraparietal (AIP) area. Cereb Cortex 18:1094-1111.

Castiello U, Begliomini C (2008) The cortical control of visually guided grasping. Neuroscientist 14:157-170.

Fluet MC, Baumann MA, Scherberger H (2010) Context-specific grasp movement representation in macaque ventral premotor cortex. J Neurosci 30:15175-15184.

Fogassi L, Gallese V, Buccino G, Craighero L, Fadiga L, Rizzolatti G (2001) Cortical mechanism for the visual guidance of hand grasping movements in the monkey: a reversible inactivation study. Brain 124:571-586.

Gerbella M, Belmalih A, Borra E, Rozzi S, Luppino G (2010) Cortical connections of the macaque caudal ventrolateral prefrontal areas $45 \mathrm{~A}$ and 45B. Cereb Cortex 20:141-168.

Gerbella M, Belmalih A, Borra E, Rozzi S, Luppino G (2011) Cortical connections of the anterior (F5a) subdivision of the macaque ventral premotor area F5. Brain Struct Funct 216:43-65.

Godschalk M, Mitz AR, van Duin B, van der Burg H (1995) Somatotopy of monkey premotor cortex examined with microstimulation. Neurosci Res 23:269-279.

Graziano MS, Taylor CS, Moore T (2002) Complex movements evoked by microstimulation of precentral cortex. Neuron 34:841-851.

Janssen P, Shadlen MN (2005) A representation of the hazard rate of elapsed time in macaque area LIP. Nat Neurosci 8:234-241.

Janssen P, Vogels R, Orban GA (1999) Macaque inferior temporal neurons are selective for disparity-defined three-dimensional shapes. Proc Natl Acad Sci U S A 96:8217-8222.

Janssen P, Vogels R, Orban GA (2000a) Selectivity for 3D shape that reveals distinct areas within macaque inferior temporal cortex. Science 288:2054-2056.

Janssen P, Vogels R, Orban GA (2000b) Three-dimensional shape coding in inferior temporal cortex. Neuron 27:385-397.

Janssen P, Srivastava S, Ombelet S, Orban GA (2008) Coding of shape and position in macaque lateral intraparietal area. J Neurosci 28:6679-6690.

Jeannerod M, Arbib MA, Rizzolatti G, Sakata H (1995) Grasping objects: the cortical mechanisms of visuomotor transformation. Trends Neurosci 18:314-320.

Joly O, Vanduffel W, Orban GA (2009) The monkey ventral premotor cortex processes 3D shape from disparity. Neuroimage 47:262-272. 
Maunsell JH, Gibson JR (1992) Visual response latencies in striate cortex of the macaque monkey. J Neurophysiol 68:1332-1344.

Murata A, Fadiga L, Fogassi L, Gallese V, Raos V, Rizzolatti G (1997) Object representation in the ventral premotor cortex (area F5) of the monkey. J Neurophysiol 78:2226-2230.

Murata A, Gallese V, Luppino G, Kaseda M, Sakata H (2000) Selectivity for the shape, size, and orientation of objects for grasping in neurons of monkey parietal area AIP. J Neurophysiol 83:2580-2601.

Nelissen K, Vanduffel W (2011) Grasping-related functional magnetic resonance imaging brain responses in the macaque monkey. J Neurosci 31:8220-8229.

Petrides M, Cadoret G, Mackey S (2005) Orofacial somatomotor responses in the macaque monkey homologue of Broca's area. Nature 435: $1235-1238$.

Premereur E, Vanduffel W, Janssen P (2011) Functional heterogeneity of macaque lateral intraparietal neurons. J Neurosci 31:12307-12317.

Rainer G, Asaad WF, Miller EK (1998) Selective representation of relevant information by neurons in the primate prefrontal cortex. Nature 393:577-579.

Raos V, Umiltá MA, Murata A, Fogassi L, Gallese V (2006) Functional properties of grasping-related neurons in the ventral premotor area F5 of the macaque monkey. J Neurophysiol 95:709-729.

Rizzolatti G, Camarda R, Fogassi L, Gentilucci M, Luppino G, Matelli M (1988) Functional organization of inferior area 6 in the macaque monkey. II. Area F5 and the control of distal movements. Exp Brain Res 71:491-507.

Rizzolatti G, Luppino G (2001) The cortical motor system. Neuron 31:889901.

Rizzolatti G, Fogassi L, Gallese V (2002) Motor and cognitive functions of the ventral premotor cortex. Curr Opin Neurobiol 12:149-154.

Sakata H, Taira M, Murata A, Mine S (1995) Neural mechanisms of visual guidance of hand action in the parietal cortex of the monkey. Cereb Cortex 5:429-438.

Sakata H, Taira M, Kusunoki M, Murata A, Tanaka Y (1997) The Trends
Neurosci Lecture. The parietal association cortex in depth perception and visual control of hand action. Trends Neurosci 20:350-357.

Srivastava S, Orban GA, De Mazière PA, Janssen P (2009) A distinct representation of three-dimensional shape in macaque anterior intraparietal area: fast, metric, and coarse. J Neurosci 29:10613-10626.

Taira M, Mine S, Georgopoulos AP, Murata A, Sakata H (1990) Parietal cortex neurons of the monkey related to the visual guidance of hand movement. Exp Brain Res 83:29-36.

Theys T, Srivastava S, van Loon J, Goffin J, Janssen P (2012) Selectivity for three-dimensional contours and surfaces in the anterior intraparietal area. J Neurophysiol 107:995-1008.

Townsend BR, Subasi E, Scherberger H (2011) Grasp movement decoding from premotor and parietal cortex. J Neurosci 31:14386-14398.

Verhoef BE, Vogels R, Janssen P (2009) The role of inferior temporal area TEs and posterior parietal area AIP in perceptual decisions about disparity-defined 3D shapes: a choice probability and inactivation study. Soc Neurosci Abstr 802.2.

Verhoef BE, Vogels R, Janssen P (2010) Contribution of inferior temporal and posterior parietal activity to three-dimensional shape perception. Curr Biol 20:909-913.

Verhoef BE, Vogels R, Janssen P (2011) Synchronization between the endstages of the dorsal and the ventral visual stream. J Neurophysiol 105:2030-2042.

Verhoef BE, Vogels R, Janssen P (2012) Inferotemporal cortex subserves three-dimensional structure categorization. Neuron 73:171-182.

Vigneswaran G, Kraskov A, Lemon RN (2011) Large identified pyramidal cells in macaque motor and premotor cortex exhibit "thin spikes": implications for cell type classification. J Neurosci 31:14235-14242.

Watt SJ, Bradshaw MF (2003) The visual control of reaching and grasping: binocular disparity and motion parallax. J Exp Psychol Hum Percept Perform 29:404-415.

Yamane Y, Carlson ET, Bowman KC, Wang Z, Connor CE (2008) A neural code for three-dimensional object shape in macaque inferotemporal cortex. Nat Neurosci 11:1352-1360. 